\title{
NOTE ON A CONJECTURE OF COXETER
}

\author{
by JOHN LEECH and JENS MENNICKE
}

(Received 16 May, 1960, and in revised form 15 August, 1960)

1. Coxeter [1] has studied groups defined by the relations

$$
\begin{aligned}
(l, m \mid n, k): A^{l} & =B^{m}=(A B)^{n}=\left(A^{-1} B\right)^{k}=E, \\
(l, m, n ; p): A^{l} & =B^{m}=(A B)^{n}=\left(A^{-1} B^{-1} A B\right)^{p}=E, \\
G^{m, n, p}: A^{m} & =B^{n}=C^{p}=(A B)^{2}=(B C)^{2}=(C A)^{2}=(A B C)^{2}=E,
\end{aligned}
$$

and gives lists of finite groups known to be completely defined by such sets of relations. In a later paper [2] he shows that $G^{3, n, p}$ is finite if $n, p$ are both even and satisfy

$$
\cos (4 \pi / n)+\cos (4 \pi / p)<\frac{1}{2}
$$

and expresses the conjecture that the restriction to even values may be removed. The only case satisfying this inequality and not already known to be finite is $G^{3,7,16}$. In this note we show that $G^{3,7,16}$ is indeed finite, being of order $21504=2^{10} .3 .7$, by showing that its subgroup

$$
(2,3,7 ; 8) \sim(8,7 \mid 2,3)
$$

of index 2 is finite and of order 10752. Thus we add one entry to each of the lists of finite groups in Coxeter [1].

These are not new finite groups. Sinkov [4] showed that the relations

$$
(8,7 \mid 2,3), \quad\left(A^{2} B^{4}\right)^{6}=E
$$

define a group of order 10752 ; our work shows that the extra relation $\left(A^{2} B^{4}\right)^{6}=E$ is redundant in this definition.

The question remains open whether there exist any finite groups $G^{3, n, p}$ not satisfying the inequality: none is known, but only those with $n, p$ even are known to be infinite [2].

2. We work with the group $(8,7 \mid 2,3)$ defined by

$$
\begin{aligned}
A^{8} & =E, \\
B^{7} & =E, \\
(A B)^{2} & =E, \\
\left(A^{-1} B\right)^{3} & =E .
\end{aligned}
$$

In this and the next section, we make no use of $(1)$; the results are therefore valid for any group satisfying (2)-(4). The relations (2)-(4) define the group $[3,7]^{+}[3$, p. 54] of direct (sensepreserving) symmetry operations of the hyperbolic tessellation $\{3,7\}$, part of which is shown in Figure 1. A number of operations of the form $A^{m} B^{n}$ are shown; there is no difficulty in supplying algebraic proofs that these have the periods implied. The axes of the hyperbolic translations $A$ and $A^{2} B^{4}$ are shown. The region of the tessellation shown is bounded by a $\{14\}$ 
(a regular 14-gon) of angle $2 \pi / 7$, one of a tessellation $\{14,7\}$ inscribed in the $\{3,7\}$. The operation $A^{4}$ takes one side of this $\{14\}$ into one five places round; similarly, if the sides are numbered consecutively, the operation $B^{-2 n} A^{4} B^{2 n}$ takes the $(2 n)$ th side into the $(2 n+5)$ th modulo 14. These operations and their inverses thus take the $\{14\}$ into any of the $\{14\} \mathrm{s}$ adjacent to it in the tessellation $\{14,7\}$, and so, by repeated application, into any $\{14\}$ of the tessellation. The operations therefore generate a normal subgroup of $[3,7]^{+}$, whose quotient group is the group of direct symmetry operations of the map obtained by identifying pairs of sides of the $\{14\}$, namely Klein's simple group of order 168 defined by the relations $(4,7 \mid 2,3)$. This normal subgroup is thus the kernel of the homomorphism between $[3,7]^{+}$and $(4,7 \mid 2,3)$.

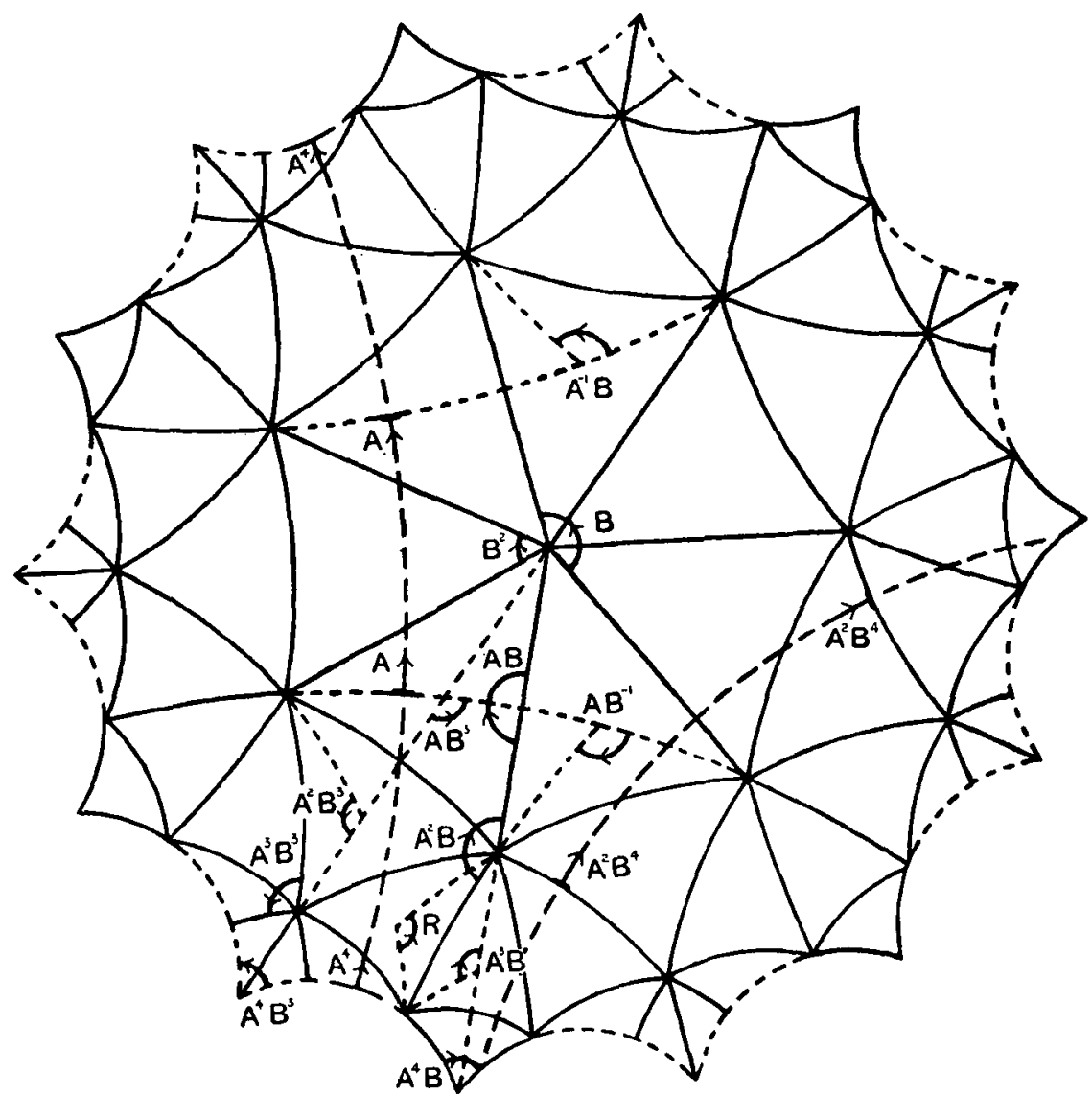

FIGURE 1

Purely algebraic proofs of this result may be obtained in two ways. One may show by enumeration of cosets $\left[3\right.$, ch. 2] that the subgroup generated by $B^{-n} A^{4} B^{n}(n=0,1, \ldots, 6)$ is of index 168; more easily one can enumerate the 24 cosets of the subgroup generated by $A^{4}, B$ and observe that the elements $B^{-n} A^{4} B^{n}$ generate a normal subgroup of index 7 in that subgroup. Alternatively one may show that any conjugate of $A^{4}$ is expressible in terms of the seven elements 


$$
\begin{aligned}
& a=A^{4}, \\
& c=B^{-1} A^{4} B, \\
& e=B^{-2} A^{4} B^{2}, \\
& g=B^{-3} A^{4} B^{3}, \\
& b=B^{-4} A^{4} B^{4}, \\
& d=B^{-5} A^{4} B^{5}, \\
& f=B^{-6} A^{4} B^{6} .
\end{aligned}
$$

This will follow if we show that for $x=a, b, c, d, e, f, g$ the element $A^{-1} x A$ is expressible in terms of $a, b, c, d, e, f, g$. In fact we find

$$
\begin{aligned}
& A^{-1} a A=a, \\
& A^{-1} b A=a^{-1} g^{-1} f^{-1}, \\
& A^{-1} c A=f, \\
& A^{-1} d A=b^{-1}, \\
& A^{-1} e A=d^{-1} f^{-1}, \\
& A^{-1} f A=a^{-1} c^{-1}, \\
& A^{-1} g A=e^{-1} .
\end{aligned}
$$

By successive application of these results we can express any conjugate of $A^{4}$ in terms of $a, b, c$, $d, e, f, g$.

3. It will be seen from Figure 1 that the operations $B^{2},\left(A^{4} B^{3}\right)^{-1}, A^{4} B$ are equal rotations, of period 7, whose centres of rotation are vertices of an equilateral triangle of angle $\pi / 7$. This triangle is one of a tessellation $\{3,14\}$ which is inscribed in the $\{3,7\}$ and in which the $\{14,7\}$ is inscribed. The operation $R=A^{2} B^{3} A^{-1}$ is a rotation of period 3 about the centre of this triangle. We thus have $R^{-1} B^{2} R=B^{-3} A^{-4}$ and $R^{-2} B^{2} R^{2}=A^{4} B$, so that conjugation by $R$ induces an outer automorphism of the group generated by $A^{4}, B$. To see that it also induces an outer automorphism of the subgroup generated by $B^{-n} A^{4} B^{n}$, we notice that the elements of this subgroup are characterized by having indices of $B$ whose sum is zero modulo 7 , and that conjugation by $R$ doubles these indices and therefore leaves this characteristic unaltered.

In particular we have

$$
\begin{aligned}
& R^{-1} a R=R^{-1}\left(A^{4} B\right)\left(B^{2}\right)^{3} R=B^{2}\left(B^{-3} A^{-4}\right)^{3}=B^{-1} A^{-4} B \cdot B^{-4} A^{-4} B^{4} \cdot A^{-4}=c^{-1} b^{-1} a^{-1}, \\
& R^{-2} a R^{2}=R^{-2}\left(A^{4} B\right)\left(B^{2}\right)^{3} R^{2}=B^{-3} A^{-4}\left(A^{4} B\right)^{3}=B^{-2} A^{4} B^{2} \cdot B^{-1} A^{4} B=e c .
\end{aligned}
$$

We also notice that

$$
a b c d e f g=\left(A^{4} B^{3}\right)^{7}=E, \quad \text { afdbgec }=\left(A^{4} B\right)^{7}=E,
$$


from which it follows that the seven elements $a, b, c, d, e, f, g$ are not independent, any six sufficing to generate the subgroup. It is however convenient to keep all seven for reasons of symmetry.

4. We now show that in the case of $(8,7 \mid 2,3)$ the seven elements $a, b, c, d, e, f, g$ generate an Abelian group of order 64. Since any conjugate of $A^{4}$ is now of period 2, we have

$$
\left(B^{-n} A^{4} B^{n}\right)^{2}=E
$$

i.e.

$$
a^{2}=b^{2}=c^{2}=d^{2}=e^{2}=f^{2}=g^{2}=E ;
$$

$$
\left(B^{-n} R^{-2} A^{4} R^{2} B^{n}\right)^{2}=E,
$$

i.e.

$$
(a c)^{2}=(b d)^{2}=(c e)^{2}=(d f)^{2}=(e g)^{2}=(f a)^{2}=(g b)^{2}=E ;
$$

$$
\left(B^{-n} R^{-1} A^{4} R B^{n}\right)^{2}=E,
$$

i.e.

$$
(a b c)^{2}=(b c d)^{2}=(c d e)^{2}=(d e f)^{2}=(e f g)^{2}=(f g a)^{2}=(g a b)^{2}=E ;
$$

together with the products

$$
\begin{aligned}
& a b c d e f g=E ; \\
& a f d b g e c=E .
\end{aligned}
$$

(7) yields

$$
\begin{aligned}
E & =a b c a b c \\
& =a b a c b c \\
& =a b a b b c b c \\
& =(a b)^{2}(b c)^{2},
\end{aligned}
$$

so that

$$
(a b)^{2}=(b c)^{-2} \text {. }
$$

By repeated application of (10) we get

$$
(a b)^{2}=(b c)^{-2}=(c d)^{2}=(d e)^{-2}=(e f)^{2}=(f g)^{-2}=(g a)^{2}=(a b)^{-2},
$$

so that

$$
(a b)^{2}=(b c)^{2}=(c d)^{2}=(d e)^{2}=(e f)^{2}=(f g)^{2}=(g a)^{2},
$$

and

(7) yields

$$
(a b)^{4}=\ldots=E .
$$

(8) yields

$a b c=c b c$,

$$
\begin{aligned}
\text { abcdefg } & =\text { cbagfed } \\
& =a b c g f e d,
\end{aligned}
$$

so that

$$
d e f g=g f e d,
$$

$$
f e d g=e f g d,
$$

giving

$$
(d g)^{2}=(e f)^{2} .
$$

$\dagger$ A reference number in the left margin indicates that the equation referred to, or a variant of it under a cyclic permutation of $a, b, c, d, e, f, g$, has been used. 
Combining (11) with (14) we get

$$
\begin{aligned}
(a b)^{2}=(b c)^{2}=(c d)^{2}=(d e)^{2} & =(e f)^{2}=(f g)^{2}=(g a)^{2} \\
& =(a d)^{2}=(b e)^{2}=(c f)^{2}=(d g)^{2}=(e a)^{2}=(f b)^{2}=(g c)^{2}
\end{aligned}
$$

(15) yields

$$
(f g)^{2}=(d g)^{2} \text {, }
$$

so that

$$
f g f=d g d
$$

and

$$
d f g=g d f \text {. }
$$

(8), (9) yield

$$
a b c d e f g=a c e g b d f \text {, }
$$

$$
a e d c b f g=a e c g b d f \text {, }
$$

$a e b c d f g=a e c b g d f$,

whence (16)

$$
b c=c b .
$$

We may therefore add " $=E$ " to (15), which with (5-8) shows that the group is Abelian and of order at most 64 . The group $(8,7 \mid 2,3)$ is therefore of order at most $168.64=10752$. Sinkov [4] gave a representation by permutations of degree 42 which shows that this is the exact order. Sinkov assumed the extra relation $\left(A^{2} B^{4}\right)^{6}=E$, which he showed to be equivalent to the relation $\left(A^{4} B^{-3} A^{4} B^{3}\right)^{2}=E$, i.e. $(a b)^{2}=E$, which we have proved above. This shows that the extra relation is redundant.

We are indebted to Professor Coxeter for comments on an earlier draft of this note. Since that draft was prepared, Dr Todd has told us that he has verified the order of $(8,7 \mid 2,3)$ by direct enumeration of the 448 cosets of the octahedral subgroup generated by $A^{2}, A^{-1} B$. This enumeration is complicated by the apparent unavoidability of having to define substantially more than that number of cosets before the working shows that these are not all distinct.

\section{REFERENCES}

1. H. S. M. Coxeter, The abstract groups $G^{m, n, p}$, Trans. Amer. Math. Soc. 45 (1939), 73-1 50.

2. H. S. M. Coxeter, Groups generated by unitary reflections of period 2, Canad.J. Math. 9 (1957), 243-272.

3. H. S. M. Coxeter and W. O. J. Moser, Generators and relations for discrete groups (Ergebnisse d. Math. N.F. 14, Springer, 1957).

4. A. Sinkov, On the group-defining relations (2, 3, 7; q), Ann. Math. (2) 38 (1937), 577-584.

THE UNIVERSITY

Glasgow 